\title{
Linear and nonlinear optical properties of $\mathrm{Si}$ nanocrystals in $\mathrm{SiO}_{2}$ deposited by plasma-enhanced chemical-vapor deposition
}

\author{
S. Hernández, ${ }^{1, a)}$ P. Pellegrino, ${ }^{1}$ A. Martínez, ${ }^{1}$ Y. Lebour, ${ }^{1}$ B. Garrido, ${ }^{1}$ R. Spano, ${ }^{2}$ \\ M. Cazzanelli, ${ }^{2}$ N. Daldosso, ${ }^{2}$ L. Pavesi, ${ }^{2}$ E. Jordana, ${ }^{3}$ and J. M. Fedeli ${ }^{3}$ \\ ${ }^{1}$ EME, Departament d'Electrónica, IN2UB, Universitat de Barcelona, Martí i Franquès 1, \\ 08028 Barcelona, Spain \\ ${ }^{2}$ Laboratorio Nanoscienze, Dipartimento di Fisica, Università di Trento, via Sommarive 14, I-38050 Povo, \\ Trento, Italy \\ ${ }^{3}$ CEA-LETI, MINATEC, 17 rue des Martyrs, 38054 Grenoble Cedex 9, France
}

(Received 27 June 2007; accepted 15 January 2008; published online 21 March 2008)

\begin{abstract}
Linear and nonlinear optical properties of silicon suboxide $\mathrm{SiO}_{x}$ films deposited by plasma-enhanced chemical-vapor deposition have been studied for different $\mathrm{Si}$ excesses up to 24 at. \%. The layers have been fully characterized with respect to their atomic composition and the structure of the $\mathrm{Si}$ precipitates. Linear refractive index and extinction coefficient have been determined in the whole visible range, enabling to estimate the optical bandgap as a function of the Si nanocrystal size. Nonlinear optical properties have been evaluated by the $z$-scan technique for two different excitations: at $0.80 \mathrm{eV}$ in the nanosecond regime and at $1.50 \mathrm{eV}$ in the femtosecond regime. Under nanosecond excitation conditions, the nonlinear process is ruled by thermal effects, showing large values of both nonlinear refractive index $\left(n_{2} \sim-10^{-8} \mathrm{~cm}^{2} / \mathrm{W}\right)$ and nonlinear absorption coefficient $\left(\beta \sim 10^{-6} \mathrm{~cm} / \mathrm{W}\right)$. Under femtosecond excitation conditions, a smaller nonlinear refractive index is found $\left(n_{2} \sim 10^{-12} \mathrm{~cm}^{2} / \mathrm{W}\right)$, typical of nonlinearities arising from electronic response. The contribution per nanocrystal to the electronic third-order nonlinear susceptibility increases as the size of the $\mathrm{Si}$ nanoparticles is reduced, due to the appearance of electronic transitions between discrete levels induced by quantum confinement. () 2008 American Institute of Physics. [DOI: 10.1063/1.2896454]
\end{abstract}

\section{INTRODUCTION}

Nanostructured silicon has generated large interest in the past decades as a promising key material to establish a $\mathrm{Si}$ based photonics. ${ }^{1}$ Room temperature light emission from $\mathrm{Si}$ nanocrystals ( $\mathrm{Si}-\mathrm{nc}$ ) is attainable by reducing their dimensions down to a few nanometers, localizing the electron-hole pairs. By controlling the size of the particles, the confinement potential can be modified and, consequently, their emission can be tuned from 1.2 to $1.75 \mathrm{eV} .^{1-5}$ On the other hand, the low absorption coefficient makes Si-nc attractive as active medium in waveguide structures. Recently, their nonlinear optical properties have been also investigated, for promising applications such as all-optical switching. ${ }^{1}$ Therefore, an accurate knowledge of both linear and nonlinear optical properties of these structures is crucial for the conception and design of highly efficient photonic structures and for the control of their performance.

Plasma-enhanced chemical-vapor deposition (PECVD) is a standard complementary metal-oxide semiconductor compatible technique, well employed to grow relatively thick $\mathrm{SiO}_{x}(x<2)$ films with a very good uniformity. ${ }^{4,5}$ The structural parameters of the precipitated $\mathrm{Si}$ nanostructures (size, distribution, density, and crystallinity) depend strongly on the deposition and annealing conditions, affecting their optical properties. On the other hand, only few works have been dedicated to the determination of the linear and nonlin-

${ }^{a)}$ Electronic mail: shernandez@el.ub.es. ear optical functions of dielectric layers containing Si-nc. ${ }^{6-9}$ So far, nonlinear refractive index $n_{2}$ of PECVD $\mathrm{SiO}_{x}$ films have been determined at $1.5 \mathrm{eV}$, reporting values of about $10^{-12} \mathrm{~cm}^{2} / \mathrm{W}$ with a large scatter in the data. ${ }^{6,7}$

In this paper, we present a systematic study of the linear and nonlinear optical properties of $\mathrm{SiO}_{x}$ films deposited on silica by PECVD technique. Several structural techniques have been used in order to determine the exact composition of the layers and the size of the Si nanoparticles. Linear optical functions have been determined as a function of the $\mathrm{Si}$ excess in the whole visible range and at $0.8 \mathrm{eV}$. By using the $z$-scan method, the nonlinear optical properties have been evaluated for two different excitation regimes: with nanosecond pulses at $0.8 \mathrm{eV}$ and with femtosecond pulses at $1.50 \mathrm{eV}$. The sign and magnitude of the third-order nonlinear susceptibility $\chi^{(3)}$ have been measured and discussed in terms of the Si excess and Si-nc size.

\section{EXPERIMENTAL DETAILS}

Substoichiometric $\mathrm{SiO}_{x}$ films were deposited by PECVD on silica substrates. A $\mathrm{N}_{2} \mathrm{O}$ flow rate was kept at 2000 SCCM (SCCM denotes cubic centimeter per minute at STP), while different flow rates of $\mathrm{SiH}_{4}$ (from 100 to $500 \mathrm{SCCM}$ ) were used in order to vary the $\mathrm{SiO}_{x}$ composition. By means of a Perkin-Elmer PHI-5500 instrument working with the $\mathrm{Al} K \alpha$ excitation line, $\mathrm{x}$-ray photoelectron spectroscopy (XPS) depth-profile measurements were per- 
TABLE I. Structural parameters of substoichiometric $\mathrm{SiO}_{x}$ films annealed at $1250{ }^{\circ} \mathrm{C}$. Composition values were evaluated by XPS along the depth. The thickness, linear refractive index, and extinction coefficient were determined by ellipsometry. The mean diameter of the precipitated Si-nc $\left(d_{\text {mean }}\right)$ was obtained by EFTEM measurements.

\begin{tabular}{cccccccc}
\hline \hline $\begin{array}{c}\text { Sample } \\
\text { code }\end{array}$ & $\begin{array}{c}\text { Composition } \\
\mathrm{SiO}_{x} \mathrm{~N}_{y}\end{array}$ & $\begin{array}{c}\mathrm{Si} \text { excess } \\
(\text { at. \% })\end{array}$ & $\begin{array}{c}d_{\text {mean }} \\
(\mathrm{nm})\end{array}$ & $\begin{array}{c}\text { Thickness }^{\mathrm{a}} \\
(\mathrm{nm})\end{array}$ & $\begin{array}{c}\text { Thickness } \\
(\mathrm{nm})\end{array}$ & $\begin{array}{c}n, k \\
\text { at } 833 \mathrm{~nm}\end{array}$ & $\begin{array}{c}n, k \\
\text { at } 1.54 \mathrm{~nm}\end{array}$ \\
\hline $\mathrm{A}$ & $\begin{array}{c}x=0.84, \\
y=0.14\end{array}$ & 24 & $4.8 \pm 1.5$ & 522 & 432 & $1.99,0.020$ & $1.95, \approx 1.6 \times 10^{-5}$ \\
$\mathrm{~B}$ & $\begin{array}{l}x=0.90, \\
y=0.17\end{array}$ & 20 & $3.9 \pm 0.8$ & 559 & 475 & $1.93,0.008$ & $1.91, \approx 1.6 \times 10^{-5}$ \\
$\mathrm{C}$ & $\begin{array}{l}x=1.36, \\
y=0.15\end{array}$ & 8 & $<1$ & 590 & 545 & $1.50,<10^{-4}$ & $1.48, \approx 1.6 \times 10^{-5}$ \\
\hline
\end{tabular}

${ }^{\mathrm{a}}$ Measured in as-deposited $\mathrm{SiO}_{x}$ films.

formed in the as-deposited films to determine the atomic composition of the layers.

After deposition, annealing treatments were carried out in a conventional furnace for $1 \mathrm{~h}$ at $T_{\text {ann }}=1250{ }^{\circ} \mathrm{C}$ under a controlled $\mathrm{N}_{2}$ flow in order to promote Si-nc precipitation. ${ }^{3-5}$ For the samples under study, previous isochronal annealing over a wide range of temperatures have revealed that this is the best treatment to form a highly dense and homogeneous distribution of Si-nc. Energy-filtered transmission electron microscopy (EFTEM) measurements were performed in the annealed samples to image the Si precipitates. The Si-nc contrast in the TEM images was enhanced by energetically filtering the electron energy-loss spectra by using a Gatan image filter (GIF 2000) around the Si plasmon $\left(E_{\mathrm{Si}}=17 \mathrm{eV}\right)$, which is well separated from the $\mathrm{SiO}_{2}$ plasmon $\left(E_{\mathrm{SiO}_{2}}\right.$ $=22 \mathrm{eV}$ ).

Room temperature photoluminescence (PL) measurements were systematically performed in all the samples using a standard lock-in technique. The $\mathrm{He}-\mathrm{Cd}$ laser line at $325 \mathrm{~nm}$ was used to pump with a power density of about $8 \mathrm{~W} / \mathrm{cm}^{2}$ on the sample. The PL signal was analyzed in backscattering configuration by a high-resolution monochromator coupled to a GaAs photomultiplier detector. The determination of the optical absorption (OA) spectrum in the range of $6.5-1.75 \mathrm{eV}$ was performed at room temperature in a Shimadzu UV-2101 system. A combination of transmission and specular reflection measurements has been used to accurately obtain the absorbance of the oxide films. Finally, the total absorbance of the films was calculated as the neperian logarithm of the intensity ratio between the incident beam (after subtraction of the reflected component) and the outgoing beam. The refractive index and extinction coefficient in the range of $2.8-1.2 \mathrm{eV}$ and the thickness of the films were obtained by a spectrophotometric ellipsometer Filmetrics F20.

Nonlinear optical properties were determined by $z$-scan measurements on Si-nc by using a Gaussian laser beam in a tight focus limiting geometry. ${ }^{6}$ Two different excitation wavelengths were used: (i) a Nd:yttrium aluminum garnet laser pumping an optical parametric oscillator working at $0.80 \mathrm{eV}$ with a $10 \mathrm{~Hz}$ repetition rate and $4 \mathrm{~ns}$ pulse duration, using peak intensities ranging from $I_{0}=10^{8}$ to $10^{9} \mathrm{~W} / \mathrm{cm}^{2}$ and (ii) a Ti:sapphire laser at $1.50 \mathrm{eV}$ with a $82 \mathrm{MHz}$ repetition rate and $70 \mathrm{fs}$ pulse duration, keeping the peak intensity at $I_{0}=1.15 \times 10^{10} \mathrm{~W} / \mathrm{cm}^{2}$. The transmission of the laser beam through the sample was recorded by a photodiode, while a continuous motor moved the sample along the optical path ( $z$ axis). An aperture was placed in front of the signal detector for closed aperture measurements. A fraction of the input beam through a beam splitter was monitored by another photodiode and the ratio of the two measured intensities was recorded as a function of the sample position $z$. The transmission with and without the aperture was measured in the far field configuration as the sample moved through the focal point, enabling the separation of the nonlinear refractive index from the nonlinear absorption. ${ }^{6}$ Taking into account the nonlinear response of our $\mathrm{SiO}_{x}$ films, the uncertainty in the determination of the nonlinear refractive index and the nonlinear absorption coefficient is about $10 \%$ for the femtosecond regime, while only about $5 \%$ for the nanosecond regime.

\section{RESULTS AND DISCUSSION}

\section{A. Structural properties}

By means of XPS, we checked that all the films display an extremely good uniformity and no oxidation of the layers occurs during the thermal treatment. A nitrogen concentration of about 8 at. \% has been detected, due to unintentional $\mathrm{N}$ incorporation during the deposition. As reported in Table I, from the relative concentration of $\mathrm{Si}, \mathrm{O}$, and $\mathrm{N}$, indicated by inclusion in square brackets, we determined the Si excess for each sample: 24 at. \% (sample A), 20 at. \% (B), and 8 at. \% (C). To avoid confusion upon the definition of Si excess, we have considered each oxygen and nitrogen atoms bonded to silicon ones (i.e., $\mathrm{SiO}_{2}$ and $\left.\mathrm{Si}_{3} \mathrm{~N}_{4}\right)$, so that $[\mathrm{Si}]_{\text {excess }}=(1$ $-0.75 y-0.5 x) /(1+x+y)$, being $x$ and $y$ the ratio $[\mathrm{O}] /[\mathrm{Si}]$ and $[\mathrm{N}] /[\mathrm{Si}]$, respectively. The assumption that each $\mathrm{N}$ atom is in the configuration corresponding to $\mathrm{Si}_{3} \mathrm{~N}_{4}$ is grounded on the value of the photoelectron binding energy $(397.7 \mathrm{eV})$, we found by XPS measurements, which indeed corresponds to such atomic arrangement. ${ }^{10}$

By ellipsometric measurements, the thickness of the films has been determined before and after annealing. After the thermal treatment, a thickness reduction has been observed of about $80-90 \mathrm{~nm}$ for samples A and B and $45 \mathrm{~nm}$ for sample $\mathrm{C}$ (see Table I). Actually, during the deposition of $\mathrm{SiO}_{x}$ films by PECVD, some $\mathrm{H}$ atoms from $\mathrm{SiH}_{4}$ are incor- 


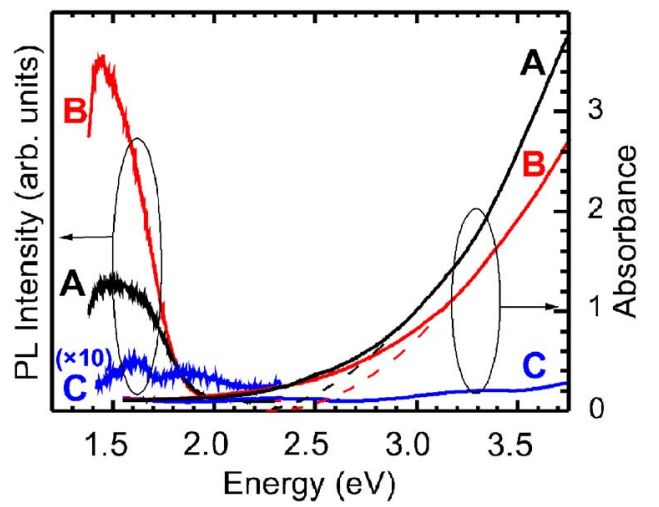

FIG. 1. (Color online) PL and OA spectra of PECVD $\mathrm{SiO}_{x}$ films after annealing at $T_{\text {ann }}=1250{ }^{\circ} \mathrm{C}$ for (A) 24 at. \%, (B) 20 at. \%, and (C) 8 at. $\% \mathrm{Si}$ excess. The dashed lines represent the $\left[(\alpha h \nu)^{1 / 2} \sim\left(h \nu-E_{\mathrm{og}}\right)\right]$ fitting to determine the optical bandgap energy.

porated in the structure creating voids and structural defects. At high annealing temperatures, an outdiffusion of $\mathrm{H}$ ions takes place and the films get more compacted. Indeed, the concentration of $\mathrm{H}$ impurities and voids in the films scales with the Si excess. Infrared spectroscopy measurements (not shown) have revealed a drastic reduction of the absorption bands related to $\mathrm{Si}-\mathrm{H}$ bonds in the spectra after annealing, corroborating the desorption of $\mathrm{H}$ atoms. ${ }^{4}$

EFTEM measurements have revealed a high density of spherical Si nanoparticles all through the annealed films, with a Gaussian size distribution. Mean sizes of 4.8 and $3.9 \mathrm{~nm}$ for samples A and B, respectively, were found (see Table I). In sample C (8 at. \% Si excess), no visible nanoparticles were detected. However, its optical behavior indicates the precipitation of $\mathrm{Si}$ nanostructures after annealing (see next section), so that we can infer that Si nanostructures with a size of about $1 \mathrm{~nm}$ (spatial resolution of the technique) or smaller have precipitated.

Similar size values $(\sim 4.2 \mathrm{~nm})$ were reported by Iacona et al. for PECVD samples with $[\mathrm{Si}]_{\mathrm{exc}}=20$ at. $\%$ and $[\mathrm{N}]$ $=9$ at. $\% .^{5}$ Interestingly, a large discrepancy is apparent in the case of Si-implanted $\mathrm{SiO}_{2}:$ a $\mathrm{Si}$ excess of 10 at. \% has been reported to give rise to nanoparticles with a mean diameter of $4 \mathrm{~nm}$ in Si-implanted $\mathrm{SiO}_{2},{ }^{11}$ while a $\mathrm{Si}$ excess around 20 at. \% is required to obtain Si-nc of similar dimensions in PECVD layers. This systematic occurrence strongly suggests a defect-enhanced Si diffusivity in ion-beam synthesized layers or, less probably, a suppression of this latter in $\mathrm{SiO}_{x} \mathrm{~N}_{y}$ matrices.

\section{B. Linear optical properties}

Room temperature PL and OA spectra are shown in Fig. 1 for the films treated at $T_{\mathrm{ann}}=1250^{\circ} \mathrm{C}$. In sample C, two weak emissions have been detected at 1.61 and $1.92 \mathrm{eV}$ related to, respectively, defective $\mathrm{SiO}_{2}$ and $\mathrm{Si}$ nanostructures. ${ }^{12}$ This is an indication that at least partial phase separation has occurred, giving rise to the luminescence characteristic of Si-nc. However, the intensity is extremely weak, suggesting a quite small nanocrystal density.

For a Si excess of 20 at. \% (spectrum B), the emission from $\mathrm{Si}$-nc shifts to lower energies $(1.44 \mathrm{eV})$ and displays an intensity enhancement of more than two orders of magnitude. In fact, this behavior is related, on one hand, to a size increase that slightly reduces the confinement potential and, on the other, to a huge increase in the particle density. By increasing the Si excess up to 24 at. \% (spectrum A), a sizable reduction of the emission intensity and a broadning of the band from the Si nc are observed in the PL spectrum. In fact, in the studied range of Si-nc sizes, the oscillator strength of the radiative recombination shrinks as the nanocrystals enlarge, producing a quenching of the PL emission. ${ }^{13}$

In Fig. 1, we also showed the absorption spectra of the suboxide films after annealing for the three different compositions. As a general trend, the absorption in the films rapidly increases toward the blue region, while it vanishes in the near-infrared range. For increasing $\mathrm{Si}$ excess, there is a progressive enhancement in the absorption of the $\mathrm{SiO}_{x}$ films, as predicted by the Bruggeman model, ${ }^{11}$ since the absorption coefficient of $\mathrm{Si}$ is higher than in $\mathrm{SiO}_{2}$. After annealing, samples A and B (24 and 20 at. \% Si excess, respectively) present a very similar absorption spectrum that scales with the Si excess: their absorption spectra are identical once normalized to the layer thickness and Si excess. This occurrence is a very strong indication of the total precipitation of the $\mathrm{Si}$ excess in form of nanoparticles, in agreement with previous observations on $\mathrm{Si}$ implanted $\mathrm{SiO}_{2} \cdot{ }^{11}$ On the contrary, sample $\mathrm{C}$ displays an absorption spectrum that does not scale with the amount of Si excess. Actually, a low Si precipitation has occurred in this sample and the contribution from suboxide phases may rule the absorption process.

We have estimated the bandgap energy of the Si-nc in each film by fitting the spectra assuming a dependence as for an indirect bandgap semiconductor $\left[(\alpha h \nu)^{1 / 2} \sim\left(h \nu-E_{\mathrm{og}}\right)\right.$, where $\alpha, h \nu$, and $E_{\mathrm{og}}$ are the absorption coefficient, the incident radiation energy, and the optical bandgap energy, respectively]. A shift from 2.72 to $2.21 \mathrm{eV}$ has been observed in the optical bandgap when the Si excess varies from 8 to 24 at. \%, attributed to a reduction in the confinement potential as the Si-nc size increases. Comparing the indirect bandgap and the emission energies, a large energy difference is observed for all the samples $(\Delta E \approx 0.6, \approx 0.8$, and $\approx 1.1 \mathrm{eV}$ for samples $\mathrm{A}, \mathrm{B}$, and $\mathrm{C}$, respectively) which is reduced for higher $\mathrm{Si}$ excesses, contrary to previous observations in Si-implanted $\mathrm{SiO}_{2}$ films where a constant energy difference $\Delta E$ of $0.26 \mathrm{eV}$ was observed independently of the $\mathrm{Si}$ content, attributed to the creation of a Si-optical phonon. ${ }^{13}$

The refractive index and extinction coefficient of the $\mathrm{SiO}_{x}$ films, as deposited and annealed, have been determined by ellipsometric measurements in the range between 1.5 and $2.75 \mathrm{eV}$. An increase of the refractive index for all the samples has been observed after thermal treatments. The high annealing temperature compacts the structure and suppress the voids while simultaneously modifies the atomic structure of the composite promoting the precipitation of Si-nc. Both effects contribute to obtain a sensible increase of the refractive index. In Fig. 2(a), we show the evolution of the refractive index with the $\mathrm{Si}$ excess for the $\mathrm{SiO}_{x}$ films after annealing. As a general trend, the refractive index in the range of study (i.e., below $2.75 \mathrm{eV}$ ) displays a smooth increase as the excitation energy approach to the $E_{0}^{\prime}$ transition 


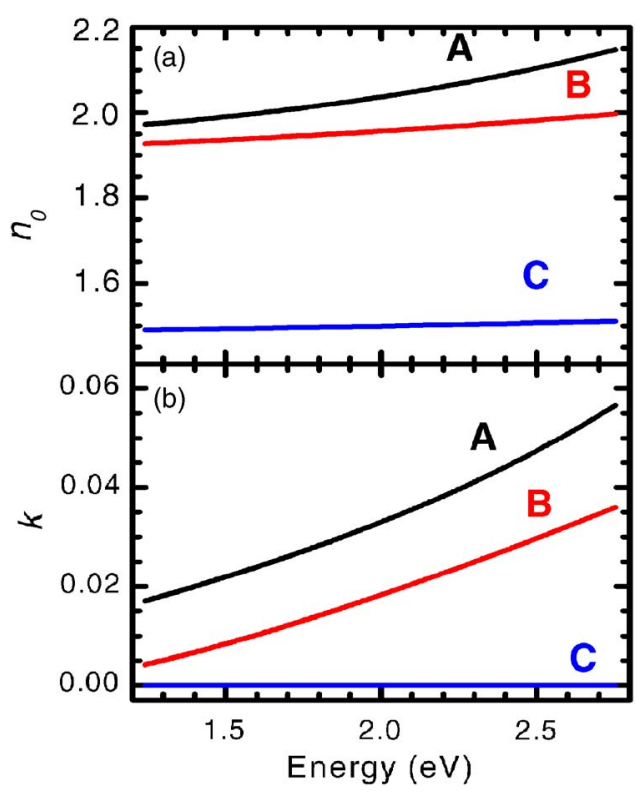

FIG. 2. (Color online) (a) Linear refractive index and (b) linear extinction coefficient of $\mathrm{SiO}_{x}$ films after an annealing at $T_{\text {ann }}=1250{ }^{\circ} \mathrm{C}$ for: (A) 24 at. $\%$, (B) 20 at. \%, and (C) 8 at. \% Si excess.

of bulk Si. On the other hand, all the samples display an increase in the refractive index with the Si excess, as expected by the higher refractive index of silicon compared to the $\mathrm{SiO}_{2}$ matrix. ${ }^{11}$

In Fig. 3, we have represented the refractive index at $1.96 \mathrm{eV}(\lambda=632 \mathrm{~nm})$ as a function of the Si excess. By using the atomic concentrations from XPS data, the dielectric functions of Si-nc (as calculated in Ref. 11), the one of $\mathrm{SiO}_{2}$ and $\mathrm{Si}_{3} \mathrm{~N}_{4}$ from literature, ${ }^{14}$ we were able to reproduce the measured refractive index with the application of the Bruggeman model. It is interesting to note the small contribution of the nitride phase to the overall $n$ value, even when the volumetric fraction of this phase gets closer to the one of crystalline $\mathrm{Si}$. The agreement between simulation and experiment, observed also for intermediated Si concentrations (not shown), is quite impressive, taking into account all the experimental uncertainties involved in such a calculation. Therefore, it validates the assumption that completed phase separation has been reached of well-separated $\mathrm{Si}_{3} \mathrm{~N}_{4}$ and crystalline $\mathrm{Si}$ complexes, these latter with the same structure and optical behavior as the ion-beam synthesized $\mathrm{Si} \mathrm{nc}$ in $\mathrm{SiO}_{2} .{ }^{11}$ This last

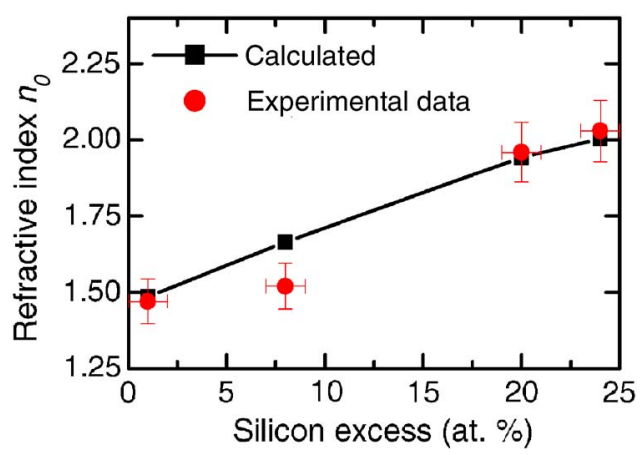

FIG. 3. (Color online) Evolution of the refractive index vs Si excess at $\lambda$ $=632 \mathrm{~nm}$. The solid line represents the simulation obtained by using a Bruggeman model which includes the $\mathrm{SiO}_{2}$ matrix and the $\mathrm{Si}$-nc and $\mathrm{Si}_{3} \mathrm{~N}_{4}$ dispersed compounds.

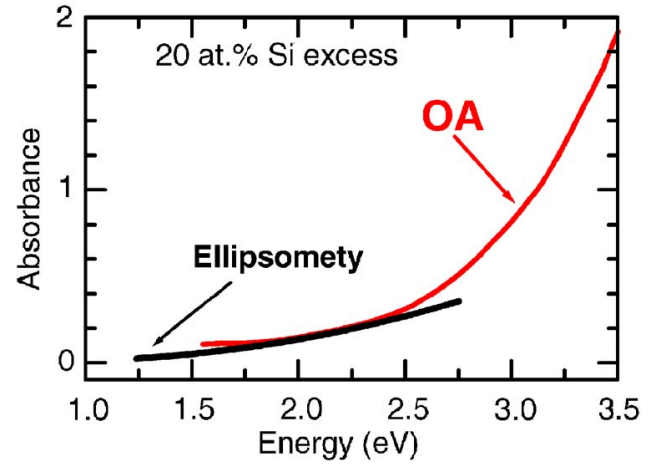

FIG. 4. (Color online) Comparison of the optical absorption spectrum (direct measurement) with the one calculated from the ellipsometric results of $k$ for sample B (20 at. \% Si excess).

assumption may not be completely true for the sample with the lowest $\mathrm{Si}$ excess, where the Bruggeman model slightly overestimates the refractive index of the layer. Once more, the experimental evidences point to a higher thermal budget required to reach a perfect $\mathrm{Si}$ precipitation in PECVD samples once the $\mathrm{Si}$ excess gets below 10 at. \%.

Figure 2(b) represents the extinction coefficient of the suboxide films after annealing. In the range between 1.25 and $2.75 \mathrm{eV}$, sample $\mathrm{C}$, both in as-deposited and annealed state, has revealed an extinction coefficient lower than the photometric accuracy of the experimental setup, i.e., $<10^{-4}$. The samples with higher $\mathrm{Si}$ excess (films A and B) have shown an increase of the extinction coefficient after annealing, similarly to what we observed in OA, where the asdeposited films presented lower absorption than after annealing. In the range of study, the extinction coefficient of these two last samples (A and B) presents a progressive increase as the energy approaches the direct bandgap transition of bulk silicon at $4.1 \mathrm{eV} .{ }^{11}$ In Fig. 4, we present a comparison of the absorption spectrum with the one from ellipsometric results for sample B (20 at. \% Si excess). An excellent agreement between the two techniques has been found, validating the fitting procedure to the reflectance data used to extract the optical functions.

In order to evaluate the magnitude of $k$ at $1.55 \mu \mathrm{m}$, too small to be detected by typical spectroscopic tools, we have measured the propagation losses in the layers by means of a prism-coupling technique, obtaining losses of about $6.5 \mathrm{~dB} / \mathrm{cm}$, independently of the sample characteristics. These findings imply an upper limit to the linear absorption coefficient of $1.3 \mathrm{~cm}^{-1}$ at this wavelength.

\section{Nonlinear optical properties}

Nonlinear transmission $z$-scan experiments of the annealed $\mathrm{SiO}_{x}$ films were systematically performed using two different excitation energies: 1.50 and $0.8 \mathrm{eV}(\lambda=830$ and $1550 \mathrm{~nm}$, respectively). Measurements on pure $\mathrm{SiO}_{2}$ have displayed no significant nonlinear optical contribution, thus, the influence of the $\mathrm{SiO}_{2}$ substrate to the $z$-scan traces could be considered negligible. Under nanosecond excitation conditions, a similar behavior has been observed in all the $\mathrm{SiO}_{x}$ films under studied. In Fig. 5, we show the $z$-scan trace for closed and open aperture configurations of sample B 


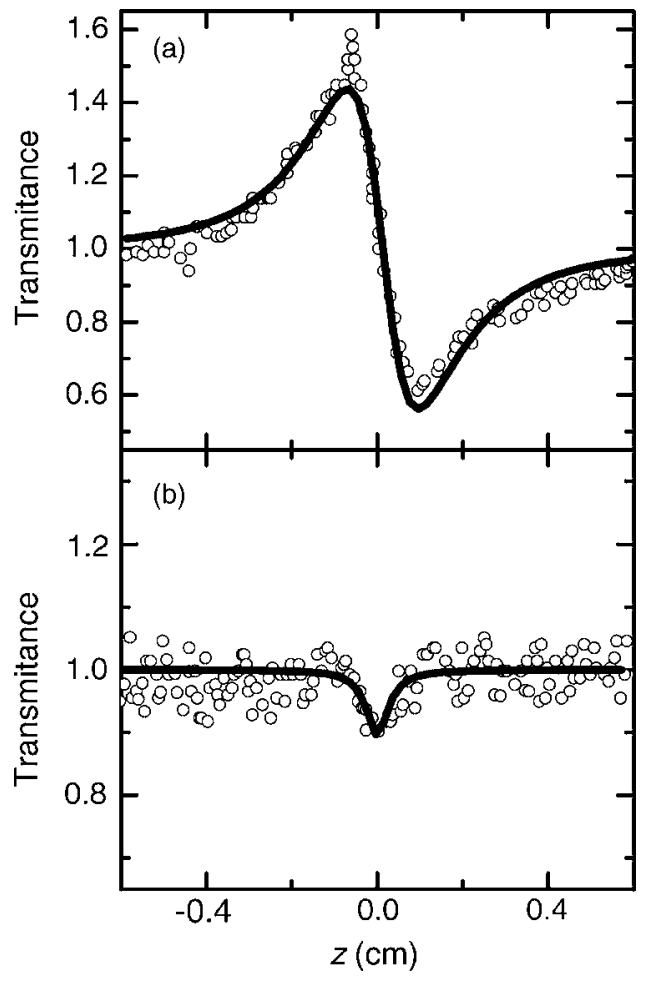

FIG. 5. Z-scan measurements of sample B annealed at $1250{ }^{\circ} \mathrm{C}$ exciting at $0.8 \mathrm{eV} \mathrm{nm}$ with $4 \mathrm{~ns}$ width pulses and $10 \mathrm{~Hz}$ repetition rate and using (a) closed aperture configuration (far field) and (b) open aperture configuration (near field). The solid lines are fitting curves using Eq. (1) for closed aperture and Eq. (2) for open aperture.

(20 at. \% Si excess), by exciting at $0.8 \mathrm{eV}$ with 4 ns pulses. Typically, the z-scan transmission in the closed aperture configuration has shown a peak-to-valley trace [see Fig. 5(a)], related to a negative nonlinear response. The duration of the pulses is long enough compared to the electronic response associated with bound electrons so that slower mechanisms, such as free carriers or thermal effects through thermal lenses, may contribute to the $z$-scan transmittance. ${ }^{6}$ On the other hand, in the open aperture configuration [Fig. 5(b)], we found a dip in the $z$-scan transmittance with a relative transmission change of about $20 \%$, showing that the nonlinear absorption is not negligible at all in our system.

To evaluate the nonlinear contributions, we have fitted the experimental transmittance $T(x)$ by the expressions ${ }^{6}$

$$
T(x)=1+4 \times \Delta \varphi /\left[\left(x^{2}+9\right)\left(x^{2}+1\right)\right],
$$

for closed aperture configuration and

$$
T(x)=1+\beta I_{0} d /\left[2 \sqrt{ } 2\left(1+x^{2}\right)\right],
$$

for open aperture configuration. In Eqs. (1) and (2), $x=z / z_{0}$ is defined as the reduce distance from the focal point, being $z$ the absolute longitudinal distance and $z_{0}$ the Rayleigh range of the beam. $I_{0}, \beta$, and $d$ are the peak intensity at the focus position, the nonlinear absorption coefficient and the thickness of the $\mathrm{SiO}_{x}$ film, respectively. Finally, the phase change $\Delta \varphi$ is related to the nonlinear refractive index $n_{2}$ by

$$
n_{2}=\Delta \varphi \lambda \alpha /\left[2 \sqrt{ } 2 \pi I_{0}\left(1-e^{-\alpha d}\right)\right],
$$

where $\alpha$ is the linear absorption coefficient at the used wavelength $\lambda$. The analysis of the $z$-scan traces results in values of

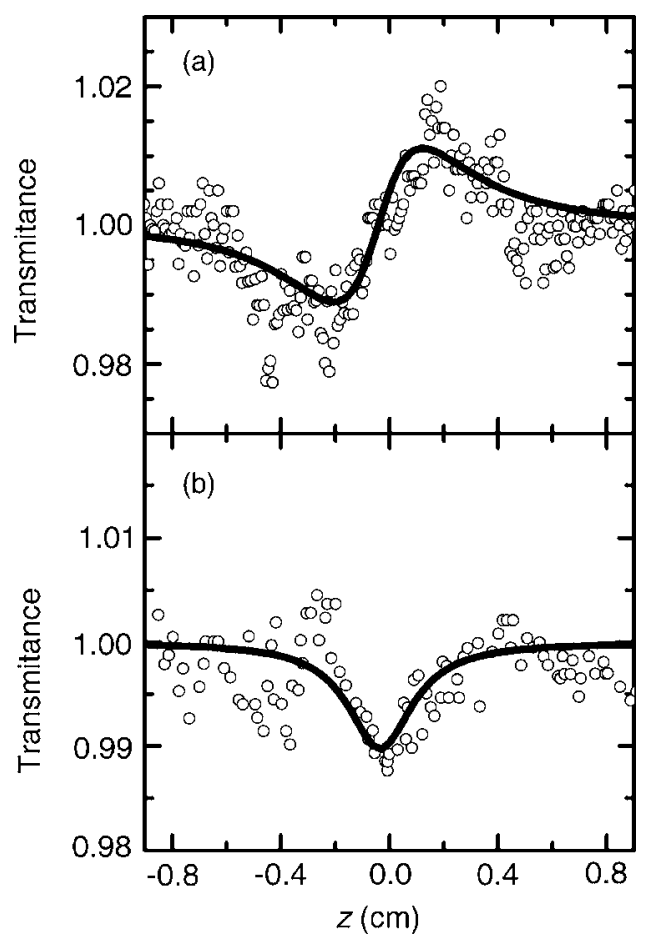

FIG. 6. Z-scan measurements of sample B annealed at $1250{ }^{\circ} \mathrm{C}$ exciting at $1.50 \mathrm{eV} \mathrm{nm}$ with $70 \mathrm{fs}$-width pulses and $82 \mathrm{MHz}$ repetition rate and using (a) closed aperture configuration (far field) and (b) open aperture configuration (near field). The solid lines are fitting curves using Eq. (1) for closed aperture and Eq. (2) for open aperture.

nonlinear refraction index $n_{2}$ from $-10^{-9}$ to $-10^{-8} \mathrm{~cm}^{2} / \mathrm{W}$ and nonlinear absorption coefficient $\beta$ from $10^{-7}$ to $10^{-6} \mathrm{~cm} / \mathrm{W}$, as the $\mathrm{Si}$ excess increases up to 24 at. $\%$. The obtained nonlinear coefficients are considerably high, leading to a nonlinear contribution $\left(n_{2}\right.$ and $\left.\beta\right)$ comparable to the linear ones ( $n$ and $\alpha$ ) for the used peak intensities.

In order to extract the contribution from bound electrons (Kerr effect) and discriminate it from thermal contributions, measurements of optical nonlinearities were performed also in femtosecond time regime at $1.50 \mathrm{eV}$, using a Ti:sapphire laser with $70 \mathrm{fs}$ pulse width and a repetition rate of $82 \mathrm{MHz}$. The experimental data for closed and open aperture configurations are shown in Fig. 6 for sample B (20 at. \%).

It is worth noting that the typical $z$-scan trace presents a reversed behavior with respect to the previously discussed case, showing a valley-to-peak shape that indicates that $n_{2}$ gets positive. Moreover, its magnitude is considerably smaller than the one obtained in the nanosecond time regime, falling around $10^{-13}-10^{-12} \mathrm{~cm}^{2} / \mathrm{W}$, typical range for electronic nonlinearities. ${ }^{6}$ We found nonlinear absorption coefficients with values around $10^{-8} \mathrm{~cm} / \mathrm{W}$, about two orders of magnitude lower than in the nanosecond regime.

In order to obtain the electronic response of the $\mathrm{SiO}_{x}$ films, the real part of the third-order nonlinear susceptibility has been calculated from $\operatorname{Re}\left[\chi^{(3)}\right]=2 n^{2} \varepsilon_{0} c n_{2}$, where $n$ is the linear refractive index, $\varepsilon_{0}$ is the permittivity of free space, and $c$ is the velocity of light in vacuum. We have plotted the real part of the third-order nonlinear susceptibility as a function of the Si excess in Fig. 7, where an increase is apparent with the amount of $\mathrm{Si}$ in the films. This behavior seems to be in contrast with previous reports of nanoparticles inside a 


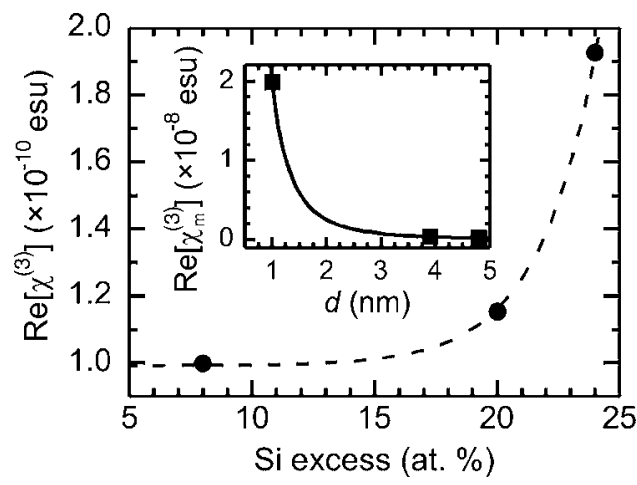

FIG. 7. Real part of the electronic susceptibility vs $\mathrm{Si}$ excess at $1.5 \mathrm{eV}$; the dashed line is a guide to the eye. In the inset, the real part of the microscopic susceptibility at the same excitation energy has been represented as a function of the Si nanocrystal size; the solid line represents the best fit to a $1 / d^{3}$ dependence.

composite, ${ }^{6}$ where an increase of $\operatorname{Re}\left[\chi^{(3)}\right]$ was observed as the average size of the nanoparticles was reduced, due to nonlinear confinement effects. However, the observed $\operatorname{Re}\left[\chi^{(3)}\right]$ accounts for an effective electronic response of the $\mathrm{SiO}_{x}$ material including, at the same time, both the density (or volume fraction) and size of the Si particles. Therefore, in order to single out the contribution of the Si aggregates, we followed the approach of Ballesteros et al. ${ }^{15}$ We considered the microscopic third-order nonlinear susceptibility $\operatorname{Re}\left[\chi_{m}^{(3)}\right]$, that is related to the effective $\operatorname{Re}\left[\chi^{(3)}\right]$ by experimentally measured linear parameters:

$$
\operatorname{Re}\left[\chi_{m}^{(3)}\right]=2 \pi / \lambda \times k_{\mathrm{Si}} /\left(f^{2} \sqrt{ } \varepsilon_{0}\right) \times \operatorname{Re}\left[\chi^{(3)}\right] / \alpha,
$$

being $f$ is the local field effect $\left[f=3 \varepsilon_{0} /\left(\varepsilon+2 \varepsilon_{0}\right)\right.$, where $\varepsilon_{0}$ and $\varepsilon$ are the dielectric constants of $\mathrm{SiO}_{2}$ and $\mathrm{Si}$, respectively], $\lambda$ the excitation wavelength and $k_{\mathrm{Si}}$, the extinction coefficient of Si. The values of $\alpha$ were obtained by ellipsometry, while $\operatorname{Re}\left[\chi^{(3)}\right]$ was obtained by $z$-scan measurements. By looking at $\operatorname{Re}\left[\chi_{m}^{(3)}\right]$, we have decorrelated the effect due to the variation of Si content between samples, and established the correct evolution of the susceptibility of a single $\mathrm{Si}$-nc versus its size. The results are shown in the inset of Fig. 7, where the experimental $\operatorname{Re}\left[\chi_{m}^{(3)}\right]$ data quite nicely follow a $1 / d^{3}$ dependence, typical of a contribution from intraband transitions induced by quantum confinement. ${ }^{15}$ The excellent agreement of our experimental data with the proposed trend shows that, in the studied size range, the main contribution to the electronic susceptibility of the Si-nc comes from intraband transitions between discrete levels in the conduction band, and the quantum confinement strongly enhances the nonlinear response of the system, mostly for Si-nc with a diameter smaller than $2 \mathrm{~nm}$.

\section{CONCLUSIONS}

We have investigated the linear and nonlinear optical properties of $\mathrm{SiO}_{x}$ films deposited by PECVD as a function of the $\mathrm{Si}$ excess. All the $\mathrm{SiO}_{x}$ films have shown an important concentration of $\mathrm{N}$ impurities (around 8 at. \%), contributing to the formation of a $\mathrm{Si}_{3} \mathrm{~N}_{4}$ phase. The structural characterization has revealed the precipitation of Si nanoclusters with a high volumetric density. A relevant thickness variation after annealing has been found as a consequence of the suppression of voids and $\mathrm{H}$ impurities. Linear optical characterization allowed to determine the emission energy, optical bandgap, and the linear optical parameters $n$ and $k$ in the whole visible range and at $0.8 \mathrm{eV}$. The overall optical functions of the films can be seen as a linear combination of the ones of the individual components: the $\mathrm{SiO}_{2}$ matrix and the Si-nc and $\mathrm{Si}_{3} \mathrm{~N}_{4}$ dispersed compounds, according to a Bruggeman model.

Nonlinear optical properties were evaluated by the $z$-scan method at two different excitation regimes: at $0.80 \mathrm{eV}$ with nanosecond pulses and at $1.50 \mathrm{eV}$ with femtosecond pulses. Under nanosecond excitation conditions, the nonlinear process is ruled by slow mechanisms, obtaining large values of both nonlinear refractive index $\left(n_{2} \sim\right.$ $\left.-10^{-8} \mathrm{~cm}^{2} / \mathrm{W}\right)$ and nonlinear absorption coefficient $(\beta$ $\left.\sim 10^{-6} \mathrm{~cm} / \mathrm{W}\right)$. Under femtosecond excitation conditions, a positive nonlinear refractive index $\left(n_{2} \sim 10^{-12} \mathrm{~cm}^{2} / \mathrm{W}\right)$ was measured, typical of nonlinearities arising from electronic response. The electronic susceptibility, associated with intraband transitions, is highly enhanced by quantum confinement for Si nc with a size below $2 \mathrm{~nm}$.

\section{ACKNOWLEDGMENTS}

We acknowledge financial support from the European Commission under the Sixth Framework (Phologic, Contract HP6-017158). S.H. acknowledges financial support from the Spanish Ministry of Science (Juan de la Cierva Program).

${ }^{1}$ N. Daldosso and L. Pavesi, Nanosilicon edited by Vijay kumar (Elsevier, New York, 2005), Chap. 1.

${ }^{2}$ P. Pellegrino, B. Garrido, C. García, J. Arbiol, J. R. Morante, M. Melchiorri, N. Daldosso, L. Pavesi, E. Scheid, and G. Sarrabayrouse, J. Appl. Phys. 97, 074312 (2005).

${ }^{3}$ B. Garrido, M. López, A. Pérez-Rodríguez, C. García, P. Pellegrino, R. Ferré, J. A. Moreno, J. R. Morante, C. Bonafos, M. Carrada, A. Claverie, J. de la Torre, and A. Souifi, Nucl. Instrum. Methods Phys. Res. B 216, 213 (2004).

${ }^{4}$ F. Iacona, G. Franzò, and C. Spinella, J. Appl. Phys. 87, 1295 (2000).

${ }^{5}$ F. Iacona, C. Bongiorno, C. Spinella, S. Boninelli, and F. Priolo, J. Appl. Phys. 95, 3723 (2004).

${ }^{6}$ G. Vijaya Prakash, M. Cazzanelli, Z. Gaburro, L. Pavesi, F. Iacona, G. Franzò, and F. Priolo, J. Appl. Phys. 91, 4607 (2002).

${ }^{7}$ G. Vijaya Prakash, M. Cazzanelli, Z. Gaburro, L. Pavesi, F. Iacona, G. Franzò, and F. Priolo, J. Mod. Opt. 49, 719 (2002).

${ }^{8}$ S. Vijayalakshmi, H. Grebel, G. Yaglioglu, R. Pino, R. Dorsinville, and C. W. White, J. Appl. Phys. 88, 6418 (2000).

${ }^{9}$ S. Vijayalakshmi, H. Grebel, Z. Iqbal, and C. W. White, J. Appl. Phys. 84, 6502 (1998).

${ }^{10}$ R. I. Hegde, P. J. Tobin, K. G. Reid, B. Maiti, and S. A. Ajuria, Appl. Phys. Lett. 66, 2882 (1995).

${ }^{11}$ J. A. Moreno, B. Garrido, P. Pellegrino, C. García, J. Arbiol, J. R. Morante, P. Marie, F. Gourbilleau, and R. Rizk, J. Appl. Phys. 98, 013523 (2005).

${ }^{12}$ D. Kovalev, H. Heckler, G. Polisski, and F. Koch, Phys. Status Solidi B 215, 871 (1999).

${ }^{13}$ B. Garrido, M. López, O. González, A. Pérez-Rodríguez, and J. R. Morante, Appl. Phys. Lett. 77, 3143 (2000).

${ }^{14} \mathrm{P}$. Yu and M. Cardona, Fundamentals of Semiconductors (Spring-Verlag, Berlin, 1996).

${ }^{15}$ J. M. Ballesteros, J. Solís, R. Serna, and C. N. Alfonso, Appl. Phys. Lett. 74, 2791 (1999). 